\title{
Failure of the first step of two-stage revision due to polymicrobial prosthetic joint infection of the hip
}

\author{
Svetlana Bozhkova $^{1} \cdot$ Rashid Tikhilov $^{1,2} \cdot$ Dmitry Labutin $^{1} \cdot$ Alexey Denisov $^{1} \cdot$ \\ Igor Shubnyakov $^{1} \cdot$ Vadim Razorenov $^{1} \cdot$ Vasilii Artyukh $^{1} \cdot$ Anna Rukina $^{1}$
}

Received: 13 November 2015 / Accepted: 26 June 2016/Published online: 7 July 2016

(c) The Author(s) 2016. This article is published with open access at Springerlink.com

\begin{abstract}
Background The unsuccessful treatment of prosthetic joint infection (PJI) with two-stage revision leads to infection recurrence. The objectives of the study were to assess the clinical and demographic characteristics of patients with polymicrobial PJI, and to evaluate the role of the microbial profile involved in PJI in the risk of infection recurrence after the first step of two-stage revision surgery.

Materials and methods A retrospective analysis of 189 cases of culture-positive PJI following total hip replacement over a 5-year period was performed. The demographic characteristics of patients, clinical symptoms, microbiology cultures of intraoperative biopsies, laboratory values of C-reactive protein (CRP), white blood cell count and erythrocyte sedimentation rate were analyzed. Patients were divided into two groups-135 with monomicrobial and 54 with polymicrobial infection.

Results Of all patients, $68.9 \%$ in the monomicrobial and $83.3 \%$ in the polymicrobial group had a body mass index $>25 \mathrm{~kg} / \mathrm{m}^{2} \quad(p=0.05)$. The median CRP values were $5.7 \mathrm{mg} / \mathrm{L}$ (IQR $4.0-10.0 \mathrm{mg} / \mathrm{L}$ ) in the monomicrobial compared to $8.8 \mathrm{mg} / \mathrm{L}$ (IQR $5.0-27 \mathrm{mg} / \mathrm{L}$ ) in the polymicrobial group $(p=0.01)$. The percentage of successful outcomes was $27.8 \%$ in patients with microbial associations $(p<0.0001)$. Gram-negative pathogens caused
\end{abstract}

Svetlana Bozhkova

clinpharm-rniito@yandex.ru

1 Vreden Russian Research Institute of Traumatology and Orthopaedics, Akademika Baikova Str., 8, 195427 St. Petersburg, Russian Federation

2 Mechnikov North-Western State Medical University, St. Petersburg, Russian Federation polymicrobial PJI in $61.5 \%$ of cases with infection recurrence (OR 4.4; $95 \%$ CI 1.18-16.37; $p=0.03$ ).

Conclusions Overweight and obese patients or those with elevated CRP had a greater risk of polymicrobial PJI. They were predisposed to recurrence of infection after the first step of two-stage revision. An unsuccessful outcome was more likely in cases with polymicrobial infection compared to those with monomicrobial infection. In addition, the presence of multidrug-resistant strains of Gram-negative bacteria substantially increased the risk of PJI treatment being unsuccessful.

Level of evidence Level III, therapeutic study.

Keywords Prosthetic joint infection - Two-stage revision . Polymicrobial PJI

\section{Introduction}

Prosthetic joint infection represents one of the most serious complications of total hip and knee arthroplasty. There have been vast improvements in operative techniques, quality of implant material and algorithms of antimicrobial therapy to prevent the onset of infection after surgery. Even though these measures appear adequate, development of PJI after total hip replacement is a clinically important issue. Its prevalence rate is estimated to be approximately $1.5 \%$ with a mortality rate of $4 \%$ within 90 days of the postoperative period [1]. The demand for joint arthroplasty is projected to grow in the coming years, thus the number of PJI cases is expected to increase [2].

Management of PJI includes several surgical procedures such as debridement and retention strategy, resection arthroplasty, arthrodesis, one-stage or two-stage exchange strategy combined with prolonged antimicrobial therapy 
and amputation of the lower limb in selected cases as a measure of last resort [3]. While most of these methods proved to be effective in the eradication of the infection, several studies demonstrated that two-stage revision protocol has a higher success rate ranging from 75-93\% [1, 4-9]. Therefore, this technique is currently considered as the gold standard in many countries [10].

The unsuccessful treatment of PJI with two-stage revision surgery leads to the recurrence of infection. This complication is usually associated with several risk factors such as age, overweight, comorbid conditions, alcohol abuse and the presence of pathogens resistant to antibiotics $[11,12]$. Gram-positive cocci are the most common causative agents in PJI. They are identified in $84 \%$ of cases, whereas the majority of them are Staphylococcus aureus and coagulase-negative staphylococci (CNS) [9]. At the same time, several studies report that Gram-negative bacteria are present in $11.5[13,14]$ and $15 \%[13,14]$ of PJI cases. These microorganisms are often associated with polymicrobial infections with a resistance to conventional antibiotic therapy. Pseudomonas aeruginosa, Escherichia coli and Klebsiella pneumoniae are frequent causative agents in this group of periprosthetic infections [15, 16]. Relapse of infection after two-stage exchange might be associated with Gram-negative bacteria. Successful outcomes of this strategy are reported to be less optimal and appear in $52 \%$ of cases [13]. Collectively, all these factors make therapy of periprosthetic infection a challenging task.

Most studies on PJI therapy with two-stage exchange present observations from the follow-up period when the surgical procedure is completed. The unsuccessful outcome of this treatment leading to recurrent infections might also occur after the first step of two-stage exchange [17]. Therefore, information about the outcomes of this step is required to improve clinical recommendations for treatment of PJI.

The objectives of our study were (1) to assess the clinical and demographic characteristics of patients with polymicrobial PJI, and to (2) evaluate the role of the microbial profile involved in PJI in the risk of infection recurrence after the first step of two-stage revision surgery.

\section{Materials and methods}

We collected and retrospectively reviewed 189 cases of culture-positive PJIs following total hip replacement. All data about patients were gathered from the medical records after approval by the Institutional Review Board.

This study covered a period of 5 years from 2008-2012. Among the patients, there were 92 males and 97 females with an overall median age of 57 years. The cohort comprised 144 cases of PJI after primary total hip arthroplasty (THA) and 45 cases after hip replacement revision surgery without any prior bone or joint infections. In all cases PJI was unilateral. All patients were treated by the three staff surgeons at the department of purulent infections at our institution.

The diagnosis of the hip PJI was confirmed by the presence of acute joint pain, a sinus tract communicating with the implant and wound dehiscence. Apart from the local symptoms, two or more positive microbiology cultures of synovial fluid aspirates or intraoperative tissue biopsies were taken into account. Laboratory parameters such as C-reactive protein (CRP), white blood cell count (WBC) and erythrocyte sedimentation rate (ESR) were also considered. In addition, the period before clinical manifestation of infection and its duration were evaluated.

All patients underwent the first step of two-stage revision which universally involves several surgical modalities such as the removal of a hip implant, debridement of infected periprosthetic tissues and subsequent insertion of a non-articulating or articulating spacer.

The initial procedure included evaluation of tissue viability in the affected area by the operating surgeon. Purulent tissues were exposed and visualized after an exploratory incision alone of the postoperative scar over the site of the implant. Up to five biopsy specimens of infected tissues and modular components of the implant were collected from each patient into sterile containers and transferred for microbiological analysis.

The next step of the procedure included ultrasonic-assisted debridement of infected tissues with the removal of the necrotic bone. Upon thorough debridement of the affected area, static spacers containing antibiotic-loaded bone cement, or articulating spacers with a metal femoral component were inserted. Bone cement (DePuy ${ }^{\circledR} \mathrm{CMW} 1$ gentamicin) contained gentamycin with the addition of 2-4 g vancomycin per $40 \mathrm{~g}$ of material. Finally, the wound was closed with sutures following insertion of 3-4 drains with active suction. In some cases, coxofemoral immobilization was applied for 3 months after the operation, particularly in patients with high risk for dislocation of the affected hip joint.

During the postoperative period, all patients received intravenous antibiotic therapy for a period of 2 weeks followed by 4-6 weeks of oral therapy. The initial antibiotic regimen included combinations of vancomycin with beta-lactam antibiotics or quinolones; alternatively, betalactam antibiotics with quinolones or aminoglycosides. After evaluation of intraoperative biopsy cultures, the therapy was corrected in accordance with the antimicrobial sensitivity of identified pathogens.

Microorganisms were isolated from homogenized intraoperative tissue biopsies and biofilms from the surface of the removed implants after sonication. Microbial species 
were identified by the staff microbiologist from cultures with the use of selective media and biochemical test panels. Patients were divided into two groups according to the microbiology reports. The group with monomicrobial infection comprised 135 patients with only one type of identified microbial species, while the polymicrobial group included 54 patients with the presence of at least two or more different species of bacteria.

The outcome of the first step of the two-stage procedure was defined as successful when patients hospitalized for reimplantation had no recurrent infection. The outcome was interpreted as unsuccessful when inflammatory signs remained or reappeared during the period between the first step and reimplantation. These signs included the presence of acute inflammation with high levels of serum CRP, development of a sinus tract and relapse or reinfection, depending on the isolated microorganisms. In all cases of infection recurrence, the old spacer was replaced with a new one. Each group of patients with either monomicrobial or polymicrobial infection was further divided according to the outcomes into categories with or without infection recurrence.

Continuous variables are presented as medians with interquartile ranges (IQR). They were assessed for normality with D'Agostino-Pearson test and compared using the nonparametric Mann-Whitney $U$ test. Categorical data are presented as counts and proportions, which were analyzed with Fisher's exact test. The association between clinical factors and successful outcomes of surgery is shown as odds ratios (OR) with $95 \%$ CI. Reported $p$ values are two-tailed. A $p$ value $<0.05$ was considered significant. All statistical tests were performed with GraphPad Prism 6.0 (CA, USA).

\section{Results}

Demographic characteristics of the patients, clinical presentation of PJI and laboratory findings are reported in Table 1. They showed no considerable difference between groups with monomicrobial and polymicrobial infection except for body mass index (BMI) and serum CRP levels. The majority of patients were overweight or obese $(73 \%, n=138)$. The median BMI was $26 \mathrm{~kg} / \mathrm{m}^{2}$ (IQR $24-28 \mathrm{~kg} / \mathrm{m}^{2}$ ) in the monomicrobial group compared to $28 \mathrm{~kg} / \mathrm{m}^{2}$ (IQR $26-30 \mathrm{~kg} / \mathrm{m}^{2}$ ) in the polymicrobial group $(p=0.01)$. Of all patients, $68.9 \%$ in the monomicrobial and $83.3 \%$ in the polymicrobial group had a BMI $>25 \mathrm{~kg} / \mathrm{m}^{2}(p=0.05)$. Variation of serum CRP levels reached significance with median values of $5.7 \mathrm{mg} / \mathrm{L}$ (IQR $4.0-10.0 \mathrm{mg} / \mathrm{L}$ ) in the monomicrobial group compared to $8.8 \mathrm{mg} / \mathrm{L}$ (IQR $5.0-27 \mathrm{mg} / \mathrm{L}$ ) in the polymicrobial group $(p=0.01)$.
According to the widely accepted classification of PJI [18], $27.4 \%$ of monomicrobial and $20.4 \%$ of polymicrobial infections in our cohort were early and occurred within 3 months after THA; 23.7 and $25.9 \%$ were delayed and occurred between 3 and 12 months after surgery, respectively. Approximately half of all cases represented late PJI which occurred at $\geq 12$ months after THA. Polymicrobial infection was identified in $28.6 \%(n=54)$ of all culturepositive PJIs $(n=189)$. Gram-positive pathogens were predominant in both groups $(p=0.02)$. S. aureus accounted for $52.6 \%$ of isolates in the monomicrobial group ( $p=0.0002$ ) (Table 2). Of all S. aureus isolates, 8.5 and $20.6 \%$ were methicillin-resistant in the monomicrobial and polymicrobial groups, respectively. Methicillinresistant Staphylococcus epidermidis (MRSE) was present in 24.3 and $31.6 \%$ of all S. epidermidis isolates in both groups. Overall, the frequency of CNS isolates in the polymicrobial group was the highest $(34.5 \%)$ among all bacterial strains. The percentage of successful outcomes after the first step of the two-stage procedure was considerably higher $(74.8 \%, n=101)$ in patients with monomicrobial infection, compared to only $27.8 \%$ $(n=15)$ in the polymicrobial group $(p<0.0001)$ (Fig. 1). Cases with microbial associations were more likely to have an unsuccessful outcome (OR 7.7; $95 \%$ CI 3.79-15.73).

Gram-negative pathogens were frequently identified in associations $(p=0.02)$. They accounted for 14.1 and $25.7 \%$ of isolates in the monomicrobial and polymicrobial groups, respectively. Non-fermenting bacteria prevailed among Gram-negative strains. Acinetobacter $s p$. and $P$. aeruginosa were identified in $7.4 \%(p=0.05)$ and $5.1 \%$ $(p=0.56)$ of isolates, respectively. The proportion of polymicrobial PJI caused by Gram-negative pathogens was $61.5 \%$ in patients with recurrent infection and only $26.7 \%$ in patients with treatment success $(p=0.03)$ (Fig. 2). The presence of Gram-negative pathogens was associated with infection recurrence (OR 4.4; $95 \%$ CI 1.18-16.37).

\section{Discussion}

The gold standard for PJI management is two-stage revision surgery with subsequent local and systemic antibiotic therapy [10]. Although many studies focus on the outcomes of the final step of the exchange procedure, unsuccessful outcome often occurs after the initial step when an antibiotic-laden spacer is inserted [17]. This study attempts to elucidate factors leading to infection recurrence after the first step of two-stage revision.

International guidelines for diagnosis and treatment of PJI recommend several sensitive diagnostic markers such as serum CRP and ESR levels [3, 19]. We found that CRP levels were significantly increased in patients with 
Table 1 Demographic, clinical and laboratory characteristics of patients with monomicrobial and polymicrobial PJI

\begin{tabular}{|c|c|c|c|}
\hline Variable & Monomicrobial infection $(n=135)$ & Polymicrobial infection $(n=54)$ & $p$ value \\
\hline Age, years & $57(49-67)$ & $57(44-69)$ & 0.84 \\
\hline $\mathrm{WBC} \times 10^{3} / \mu \mathrm{L}$ & $8.1(6.2-9.9)$ & $7.3(5.5-9.1)$ & 0.1 \\
\hline BMI, $\mathrm{kg} / \mathrm{m}^{2}$ & $26(24-28)$ & $28(26-30)$ & 0.01 \\
\hline$\leq 25, n$ & $42(31.1 \%)$ & $9(16.7 \%)$ & 0.05 \\
\hline$>25, n$ & $93(68.9 \%)$ & $45(83.3 \%)$ & \\
\hline $\mathrm{ESR}, \mathrm{mm} / \mathrm{h}$ & $25(15-40)$ & $26(15-49)$ & 0.64 \\
\hline$\leq 30, n$ & $85(63.0 \%)$ & $31(57.4 \%)$ & 0.51 \\
\hline$>30, n$ & $50(37.0 \%)$ & $23(42.6 \%)$ & \\
\hline $\mathrm{CRP}, \mathrm{mg} / \mathrm{L}$ & $5.7(4.0-10.0)$ & $8.8(5.0-27)$ & 0.01 \\
\hline$\leq 10, n$ & $101(74.8 \%)$ & $31(57.4 \%)$ & 0.02 \\
\hline$>10, n$ & $34(25.2 \%)$ & $23(42.6 \%)$ & \\
\hline PJI after prior revision surgery, $n$ & $34(25.1 \%)$ & $11(20.4 \%)$ & 0.57 \\
\hline Manifestation of infection, days & $365(90-1500)$ & $600(120-1575)$ & 0.76 \\
\hline Pain, $n$ & $134(99.3 \%)$ & $54(100 \%)$ & 1.0 \\
\hline Sinus tract, $n$ & $100(74.1 \%)$ & $46(85.2 \%)$ & 0.13 \\
\hline Wound dehiscence, $n$ & $1(0.7 \%)$ & 0 & 1.0 \\
\hline Duration of infection, days & $150(60-300)$ & $165(60-400)$ & 0.47 \\
\hline Duration of surgery, min & $190(160-240)$ & $200(171-230)$ & 0.62 \\
\hline Blood loss, mL & $800(600-1200)$ & $900(600-1200)$ & 0.71 \\
\hline Drainage blood loss, $\mathrm{mL}$ & $250(140-350)$ & $300(200-300)$ & 0.70 \\
\hline With articulating spacer, $n$ & $73(54.1)$ & $24(44.4)$ & 0.26 \\
\hline \multicolumn{4}{|l|}{ Concomitant pathology } \\
\hline Cardiovascular pathology, $n$ & $99(73.3 \%)$ & $36(66.7 \%)$ & 0.37 \\
\hline Diabetes mellitus, $n$ & $27(20.0 \%)$ & $11(20.4 \%)$ & 1.0 \\
\hline COPD, $n$ & $24(17.8 \%)$ & $8(14.8 \%)$ & 0.68 \\
\hline \multicolumn{4}{|l|}{ Indication for THA } \\
\hline Osteoarthritis, $n$ & $80(59.3 \%)$ & $39(72.2 \%)$ & 0.13 \\
\hline Secondary osteoarthritis with rheumatoid arthritis, $n$ & $12(8.9 \%)$ & $2(3.7 \%)$ & 0.36 \\
\hline Femoral head fracture, $n$ & $19(14.1 \%)$ & $5(9.3 \%)$ & 0.47 \\
\hline Avascular necrosis of the femoral head, $n$ & $24(17.8 \%)$ & $8(14.8 \%)$ & 0.68 \\
\hline
\end{tabular}

All continuous variables are presented as medians with IQR

polymicrobial infection reaching a median of $8.8 \mathrm{mg} / \mathrm{L}$. This was in agreement with suggested prognostic criteria but below the diagnostic value of $10 \mathrm{mg} / \mathrm{L}$ for periprosthetic infection after THA [20-23]. The median ESR level was below the suggested value of $30 \mathrm{~mm} / \mathrm{h}$ with no significant difference between study groups. We believe that the low activity of these inflammatory markers in our patients was due to the chronic state of PJI.

Concomitant somatic conditions are usually considered an important risk factor of PJI [11, 12]. In our study, the incidence of cardiovascular pathology and diabetes mellitus was high in both groups but polymicrobial PJI occurred regardless of these predisposing conditions. Although the majority of our patients were either overweight or obese with a BMI $>25 \mathrm{~kg} / \mathrm{m}^{2}, 83.3 \%(n=45)$ of them had polymicrobial infection. Thus, these are the factors associated with the development of PJI which particularly increase the chance of polymicrobial infection [24]. Patients with a BMI $>25 \mathrm{~kg} / \mathrm{m}^{2}$ had a greater risk of infection caused by microbial associations (OR 2.3; CI $95 \%$ 1.01-5.04).

In a case-control study, Berbari et al. [25] reported that patients who had arthroplasty prior to THA or total knee arthroplasty were under a great risk of PJI. As depicted in Table 1, the majority of our patients had PJI after primary THA. The proportion of these patients with infection recurrence after the first step of the two-stage procedure was higher than those with prior revision arthroplasty. Presumably, this happened due to the long duration of PJI, i.e., from onset until the removal of the prosthesis. The 
Table 2 Variety of pathogens isolated from patients with monomicrobial and polymicrobial PJI

\begin{tabular}{|c|c|c|c|}
\hline Pathogens & Monomicrobial infection $n(\%)$ & Polymicrobial infection $n(\%)$ & $p$ value \\
\hline All Gram-positive & $116(85.9)$ & $101(74.3)$ & 0.02 \\
\hline S. aureus/MRSA & $71 / 6 *\left(52.5 / 8.5^{*}\right)$ & $34 / 7 *\left(25 / 20.6^{*}\right)$ & $<0.01 / 0.11$ \\
\hline S. epidermidis/MRSE & $37 / 9 * *(20.1 / 24.3 * *)$ & $38 / 12 * *(27.9 / 31.6 * *)$ & $0.15 / 1.0$ \\
\hline Other CNS & $4(3.0)$ & $9(6.6)$ & 1.0 \\
\hline Enterococcus sp. & $6(4.4)$ & $11(8.1)$ & 1.0 \\
\hline Streptoccus spp. & $4(3.0)$ & $6(4.4)$ & 1.0 \\
\hline Propionibacterium acnes & $2(1.5)$ & $6(4.4)$ & 0.71 \\
\hline Corynebacterium spp & $2(1.5)$ & $3(2.2)$ & 1.0 \\
\hline Other Gram-positive & $4(3.0)$ & $3(2.2)$ & 0.41 \\
\hline All Gram-negative & $19(14.1)$ & $35(25.7)$ & 0.02 \\
\hline Enterobacteriaceae & $7(5.1)$ & $13(9.6)$ & 0.16 \\
\hline Escherichia coli & $3(2.2)$ & $6(4.4)$ & 0.31 \\
\hline Klebsiella pneumoniae & $1(0.7)$ & $2(1.5)$ & 0.6 \\
\hline Klebsiella pneumoniae (ESBLs) & $1(0.7)$ & $3(2.2)$ & 0.35 \\
\hline Enterobacter cloacae & $2(1.5)$ & $2(1.5)$ & 1.0 \\
\hline Serratia marcescens & $2(1.5)$ & $1(0.7)$ & 1.0 \\
\hline Providencia sp. & 0 & $1(0.7)$ & 0.47 \\
\hline Aeromonas sp. & 0 & $1(0.7)$ & 0.47 \\
\hline Alcaligenes sp. & 0 & $1(0.7)$ & 0.47 \\
\hline Acinetobacter sp. & $3(2.2)$ & $10(7.4)$ & 0.045 \\
\hline Pseudomonas aeruginosa & $5(3.7)$ & $7(5.1)$ & 0.56 \\
\hline Stenotrophomonas & $1(0.7)$ & $1(0.7)$ & 1.0 \\
\hline Actinobacillus & $1(0.7)$ & 0 & 1.0 \\
\hline Candida sp. & 0 & $1(0.7)$ & 0.47 \\
\hline Total & 135 & 136 & \\
\hline
\end{tabular}

Values are presented as the number of isolates. Data were analyzed with Fisher's exact test

* Number (\%) of all S. aureus in the group; ** number (\%) of all S. epidermidis in the group
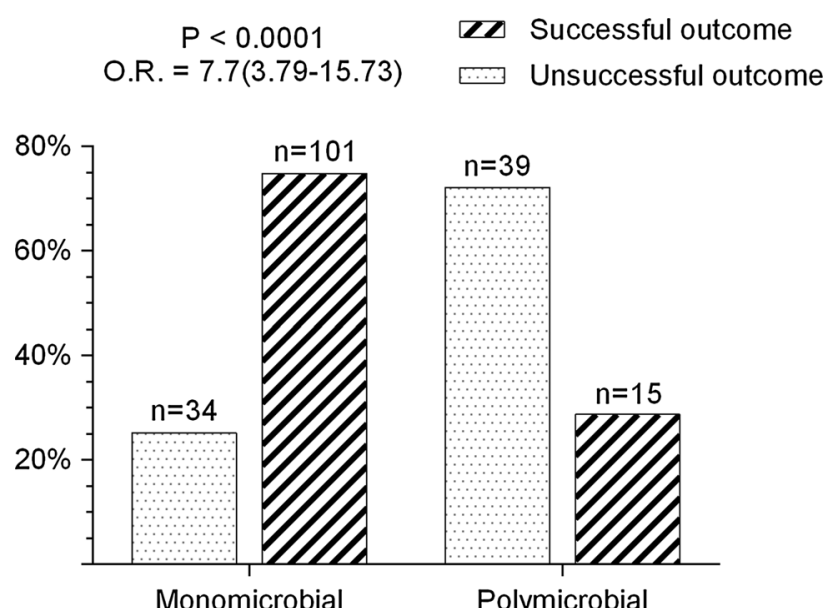

Fig. 1 Percentage of cases with successful and unsuccessful outcomes in monomicrobial and polymicrobial PJI

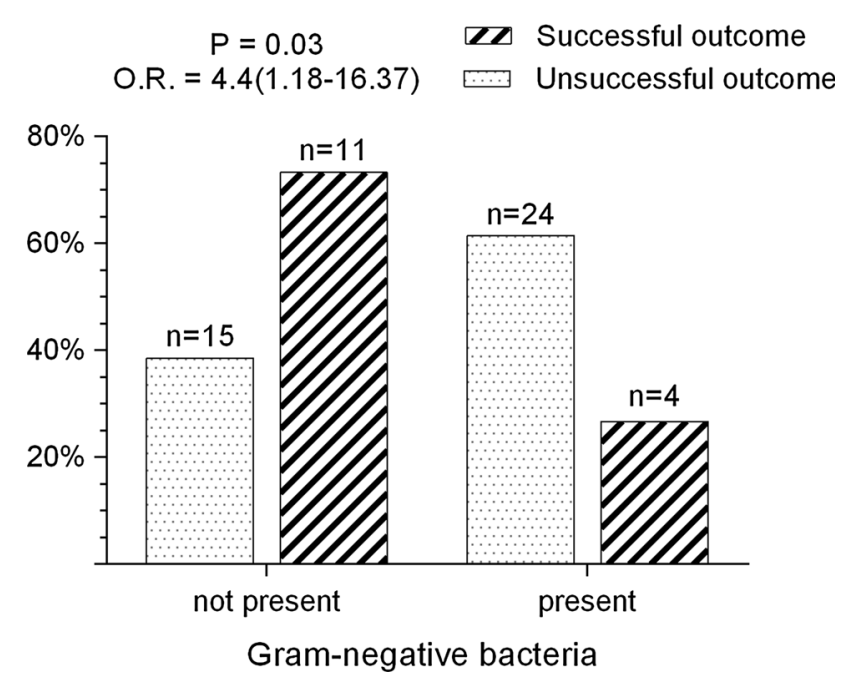

Fig. 2 Percentage of cases with successful and unsuccessful outcomes in polymicrobial PJI depending on the presence of Gramnegative pathogens in microbial associations 
median time period was 150 (IQR 60-300) and 160 (IQR 60-400) days in the monomicrobial and polymicrobial groups, respectively.

In our study, polymicrobial infection was diagnosed in $28.6 \%$ cases of PJI after THA which is close to the range of 19-37\% reported elsewhere [26, 27]. These authors also showed that polymicrobial PJIs occur more often in the early postoperative period. We did not observe any significant difference in manifestation time of infection between study groups. The median time period was 365 days for monomicrobial PJI and 600 days for polymicrobial infection $(p=0.76)$. The majority of our PJI cases were either delayed or late infections.

Polymicrobial infection is traditionally considered a risk factor for failure of one-stage revision in the management of PJI presenting a contraindication for this type of surgery $[19,28]$. The cumulative probability of PJI treatment success with surgical revision reported by Marculescu et al. [26] was $63.8 \%$ for cases with polymicrobial PJI and $72.8 \%$ for those with monomicrobial infection. The authors showed that the 2-year survival rate without infection after the two-stage procedure was 83.9 and $77.7 \%$ for monomicrobial and polymicrobial PJI, respectively. The difference was statistically insignificant due to the small number of observations (49 and 9 cases of monomicrobial and polymicrobial infection, respectively). Comparable rates were also reported by Wimmer et al. [29] in a cohort of 77 cases of total hip and knee arthroplasty, where $87.5 \%$ of their patients with monomicrobial compared to $67.6 \%$ with polymicrobial PJI were free of infection. In our study, the outcome was defined after the first step of the two-stage procedure. Although our results could not be directly compared to the above reports due to this discrepancy, we found a similar rate of success $(74.8 \%)$ in monomicrobial PJIs, but a lower $(27.8 \%)$ rate in cases with polymicrobial infection. Moreover, cases with microbial associations in our cohort were more likely to result in infection recurrence (OR 7.7; CI 95\%, 3.79-15.73).

Gram-positive bacteria were dominant in both study groups with Staphylococcus sp. being the most frequent pathogens (Table 2). Some authors indicate that the presence of methicillin-resistant strains predicts an unsuccessful outcome of PJI [11]. In our study, identification of these strains was not associated with unsuccessful outcomes of the first step of two-stage revision. In the group with monomicrobial infection, the proportion of methicillin-resistant strains in patients with unsuccessful and successful outcomes was 8.7 and $17.3 \%$, respectively. Similar findings were found in the polymicrobial group with 23.6 and $35.3 \%$ of all staphylococci, respectively. This might be due to (a) incorporation of vancomycin into a gentamicinloaded cement spacer, or (b) administration of empiric antimicrobial therapy that always included vancomycin combined with beta-lactams or quinolones in patients with negative cultures of aspirates or without any preoperative results. As a result, initial local and systemic antimicrobial therapy was effective against Gram-positive bacteria including both methicillin-sensitive and methicillin-resistant strains of staphylococci. As evident from our data, $79.3 \%$ of patients with Gram-positive monomicrobial PJI had sustained remission of the infection.

In most cases, antibiotics effective against multidrugresistant (MDR) strains of Gram-negative bacteria (extended-spectrum beta-lactamase [ESBL]-producing $K$. pneumonia, Acinetobacter $s p$. and $P$. aeruginosa) were administered only after isolation of these pathogens from intraoperative cultures of infected tissues and/or removed components of the implant. In addition, gentamicin which is released from bone cement has low activity against this type of pathogens. Therefore, ineffective initial therapy with local antibiotics present in cement spacers and systemic antimicrobial regimens might have led to an unsuccessful outcome of PJI. Of nine patients with monomicrobial PJI, caused by Gram-negative MDR strains, only one patient had sustained remission. Of 18 patients with polymicrobial infection, caused by microbial associations with MDR strains, the first step of the twostage procedure was also successful in one patient.

Our study had several limitations. Firstly, this is a single-center study. Due to its retrospective nature, it was not always possible to collect a complete case history. In particular, other concomitant conditions that might be a risk for PJI or details regarding previous arthroplasties were not always available. Secondly, the initial antibiotic therapy in our cohort was varied. There were patients who received either empirical or causative therapy. Thirdly, there might have been slight deviations in the surgical technique of two-stage revision because not every case was managed by the same operating surgeon. Finally, we did not follow-up patients with an unsuccessful outcome after replacement of the old spacer with a new one. Considering the difficulty in collecting an appreciable number of cases with PJI for a study, the relatively large number of patients $(n=189)$ in our cohort should be sufficient to overcome potential bias.

In conclusion, polymicrobial infection was observed in $28.6 \%$ of all PJI cases. Approximately half of all cases presented with late PJI which occurred at $\geq 12$ months after THA. We found that overweight and obese patients or those with elevated CRP levels had a greater risk of polymicrobial PJI and hence they were predisposed to recurrence of infection. Cases that presented with polymicrobial infection were more likely to result in an unsuccessful outcome after surgery $(72.2 \%)$ compared to those with monomicrobial infection $(25.2 \%)$. In addition, 
the presence of MDR strains of Gram-negative bacteria substantially increased the risk of failure for this type of surgery in both study groups. Despite the fact that leading pathogens in both the monomicrobial and polymicrobial groups were staphylococci, cases of PJI where empirical antibacterial therapy was administered required local and systemic antimicrobial combinations that are active against MDR strains of Gram-negative bacteria. We conclude that it is necessary to perform further multicenter prospective studies on polymicrobial PJI in a larger cohort of patients in order to identify other risk factors and develop effective measures to reduce the chance of adverse outcomes.

Acknowledgments This study was supported by the grant $N^{0} 115030510010$ from the Ministry of Health of the Russian Federation.

\section{Compliance with ethical standards}

Conflict of interest The authors declare that they have no conflict of interest.

Ethical standards The study was approved by the Institutional Review Board. Obtaining the informed consent from involved patients was waived by the Institutional Review Board. All procedures involving human participants were in accordance with the 1964 Helsinki declaration and its later amendments.

Open Access This article is distributed under the terms of the Creative Commons Attribution 4.0 International License (http://crea tivecommons.org/licenses/by/4.0/), which permits unrestricted use, distribution, and reproduction in any medium, provided you give appropriate credit to the original author(s) and the source, provide a link to the Creative Commons license, and indicate if changes were made.

\section{References}

1. Berend KR, Lombardi AV, Morris MJ, Bergeson AG, Adams JB, Sneller MA (2012) Two-stage treatment of hip periprosthetic joint infection is associated with a high rate of infection control but high mortality. Clin Orthop Rel Res 471:510. doi:10.1007/ s11999-012-2595-x

2. Kurtz S (2007) Projections of primary and revision hip and knee arthroplasty in the United States from 2005 to 2030. J Bone Joint Surg Am 89:780. doi:10.2106/jbjs.f.00222

3. Osmon DR, Berbari EF, Berendt AR, Lew D, Zimmerli W, Steckelberg JM, Rao N, Hanssen A, Wilson WR (2012) Diagnosis and management of prosthetic joint infection: clinical practice guidelines by the Infectious Diseases Society of America. Clin Infect Dis 56:e1-e25. doi:10.1093/cid/cis803

4. Choi H-R, Kwon Y-M, Freiberg AA, Malchau H (2013) Comparison of one-stage revision with antibiotic cement versus twostage revision results for infected total hip arthroplasty. J Arthroplasty 28:66-70. doi:10.1016/j.arth.2013.02.037

5. ECDC (2012) European Centre for Disease Prevention and Control. Title. Surveillance of surgical site infections in Europe, 2008-2009. Stockholm. doi: 10.2900/21096

6. Tsaras G, Osmon DR, Mabry T, Lahr B, Saint Sauveur J, Yawn B, Kurland R, Berbari EF (2012) Incidence, secular trends and outcomes of prosthetic joint infection (PJI): a population based study, olmsted county, minnesota, 1969-2007. Infect Control Hosp Epidemiol 33:1207-1212. doi:10.1086/668421

7. Dieckmann R, Schulz D, Gosheger G, Becker K, Daniilidis K, Streitbürger A, Hardes J, Hoell S (2014) Two-stage hip revision arthroplasty with a hexagonal modular cementless stem in cases of periprosthetic infection. BMC Musculoskelet Disord 15:398. doi:10.1186/1471-2474-15-398

8. Lamagni T (2014) Epidemiology and burden of prosthetic joint infections. J Antimicrob Chemother 69:i5-i10. doi:10.1093/jac/ dku247

9. Peel TN, Cheng AC, Buising KL, Choong PFM (2012) Microbiological aetiology, epidemiology, and clinical profile of prosthetic joint infections: are current antibiotic prophylaxis guidelines effective? Antimicrob Agents Chemother 56:2386-2391. doi:10.1128/AAC.06246-11

10. Cooper HJ, Valle Della CJ (2013) The two-stage standard in revision total hip replacement. Bone Joint J 95-B:84-87. doi:10. 1302/0301-620x.95b11.32906

11. Spiegl U, Friederichs J, Pätzold R, Militz M, Josten C, Bühren V (2012) Risk factors for failed two-stage procedure after chronic posttraumatic periprosthetic hip infections. Arch Orthop Trauma Surg 133:421-428. doi:10.1007/s00402-012-1673-6

12. Wu C, Qu X, Liu F, Li H, Mao Y, Zhu Z (2014) Risk factors for periprosthetic joint infection after total hip arthroplasty and total knee arthroplasty in chinese patients. PLoS One 9:e95300. doi:10.1371/journal.pone.0095300

13. Zmistowski B, Fedorka CJ, Sheehan E, Deirmengian G, Austin MS, Parvizi J (2011) Prosthetic joint infection caused by gramnegative organisms. J Arthroplasty 26:104-108. doi:10.1016/j. arth.2011.03.044

14. Hsieh P-H, Lee MS, Hsu K-Y, Chang Y-H, Shih H-N, Ueng SW (2009) Gram-negative prosthetic joint infections: risk factors and outcome of treatment. Clin Infect Dis 49:1036-1043. doi:10. $1086 / 605593$

15. Fernandes A, Dias M (2013) The microbiological profiles of infected prosthetic implants with an emphasis on the organisms which form biofilms. J Clin Diagn Res 7:219-223. doi:10.7860/ JCDR/2013/4533.2732

16. Rodríguez-Pardo D, Pigrau C, Lora-Tamayo J, Soriano A, del Toro MD, Cobo J, Palomino J, Euba G, Riera M, Sánchez-Somolinos M, Benito N, Fernández-Sampedro M, Sorli L, Guio L, Iribarren JA, Baraia-Etxaburu JM, Ramos A, Bahamonde A, Flores-Sánchez X, Corona PS, Ariza J (2014) Gram-negative prosthetic joint infection: outcome of a debridement, antibiotics and implant retention approach. A large multicentre study. Clin Microbiol Infect 20:O911-O919. doi:10.1111/1469-0691.12649

17. de Sanctis J, Teixeira L, van Duin D, Odio C, Hall G, Tomford JW, Perez F, Rudin SD, Bonomo RA, Barsoum WK, Joyce M, Krebs V, Schmitt S (2014) Complex prosthetic joint infections due to carbapenemase-producing Klebsiella pneumoniae: a unique challenge in the era of untreatable infections. Int $\mathrm{J}$ Infect Dis 25:73-78. doi:10.1016/j.ijid.2014.01.028

18. Zimmerli W, Trampuz A, Ochsner PE (2004) Prosthetic-joint infections. N Engl J Med 351:1645-1654. doi:10.1056/ nejmra040181

19. Valle Della C, Parvizi J, Bauer TW, DiCesare PE, Evans RP, Segreti J, Spangehl M, Watters WC, Keith M, Turkelson CM, Wies JL, Sluka P, Hitchcock K (2011) American academy of orthopaedic surgeons clinical practice guideline on: the diagnosis of periprosthetic joint infections of the hip and knee. J Bone Joint Surg 93:1355-1357. doi:10.2106/jbjs.9314ebo

20. Bernard L, Lübbeke A, Stern R, Bru JP, Feron JM, Peyramond D, Denormandie P, Arvieux C, Chirouze C, Perronne C, Hoffmeyer P, L'Ostéite GDS (2004) Value of preoperative investigations in diagnosing prosthetic joint infection: retrospective cohort study 
and literature review. Scand J Infect Dis 36:410-416. doi:10. 1080/00365540410015240

21. Schinsky MF (2008) Perioperative testing for joint infection in patients undergoing revision total hip arthroplasty. J Bone Joint Surg Am 90:1869-1875. doi:10.2106/JBJS.G.01255

22. Ghanem E, Antoci V, Pulido L, Joshi A, Hozack W, Parvizi J (2009) The use of receiver operating characteristics analysis in determining erythrocyte sedimentation rate and C-reactive protein levels in diagnosing periprosthetic infection prior to revision total hip arthroplasty. Int J Infect Dis 13:e444-e449. doi:10.1016/ j.ijid.2009.02.017

23. Yuan K, Chen H-L, Cui Z-M (2014) Diagnostic accuracy of C-reactive protein for periprosthetic joint infection: a metaanalysis. Surg Infect 15:548-559. doi:10.1089/sur.2013.066

24. Dowsey MM, Choong PFM (2008) Obesity is a major risk factor for prosthetic infection after primary hip arthroplasty. Clin Orthop Relat Res 466:153-158. doi:10.1007/s11999-007-0016-3

25. Berbari EF, Hanssen AD, Duffy MC, Steckelberg JM, Ilstrup DM, Harmsen WS, Osmon DR (1998) Risk factors for prosthetic joint infection: case-control study. Clin Infect Dis 27:1247-1254. doi: $10.1086 / 514991$
26. Marculescu CE, Cantey JR (2008) Polymicrobial prosthetic joint infections: risk factors and outcome. Clin Orthop Rel Res 466:1397-1404. doi:10.1007/s11999-008-0230-7

27. Moran E, Masters S, Berendt AR, Mclardy-Smith P, Byren I, Atkins BL (2007) Guiding empirical antibiotic therapy in orthopaedics: the microbiology of prosthetic joint infection managed by debridement, irrigation and prosthesis retention. J Infect 55:1-7. doi:10.1016/j.jinf.2007.01.007

28. Jackson WO, Schmalzried TP (2000) Limited role of direct exchange arthroplasty in the treatment of infected total hip replacements. Clin Orthop Rel Res 381:101-105. doi:10.1097/ 00003086-200012000-00012

29. Wimmer MD, Friedrich MJ, Randau TM, Ploeger MM, Schmolders J, Strauss AA, Hischebeth GTR, Pennekamp PH, Vavken $\mathrm{P}$, Gravius S (2015) Polymicrobial infections reduce the cure rate in prosthetic joint infections: outcome analysis with two-stage exchange and follow-up $\geq$ two years. Int Orthop. doi:10.1007/ s00264-015-2871-y 